\title{
Magnetic reconnection in 2D stratified atmospheres
}

\section{Dynamical consequences}

\author{
K. Galsgaard ${ }^{1}$ and I. Roussev ${ }^{2,3}$ \\ 1 University of St. Andrews, School of Mathematics and Statistics, North Haugh, St. Andrews, Fife, KY16 9SS, \\ Scotland, UK \\ e-mail: klaus@mcs.st-and.ac.uk \\ 2 High Altitude Observatory, NCAR, 3450 Mitchell Lane, Boulder, CO 80301, USA \\ 3 Armagh Observatory, College Hill, Armagh, BT61 9DG, N. Ireland
}

Received 1 August 2001 / Accepted 4 December 2001

\begin{abstract}
We explore the dynamical consequences of magnetic reconnection in a $2 \mathrm{D}$ stratified physical configuration representing a "quiet" solar environment. By including gravity, an initial magneto-hydrostatic solution is found that allows the magnetic field to expand with height. The change in kinetic gas pressure with height leads to the formation of a cold current "sheet" in the case of strong stratification, in contrast to a hot current "sheet" in the case of negligible stratification. Here the "sheet" temperature is measured relative to the temperature in the ambient background plasma. The dynamics of magnetic reconnection in a stratified atmosphere evolves through a new initial stage, with a more complex velocity structure than the quadrupolar velocity pattern present in traditional 2D $X$-point reconnection. As time progresses, the new initial phase is suppressed and the driven reconnection evolves into the traditional $2 \mathrm{D}$ reconnection pattern. The transition time between the two regimes is found to depend on the imposed stratification, and through this, on the degree of expansion of the initial magnetic field with height. The new reconnection regime undergoes a more complicated physical evolution and seems to have a lower reconnection rate than the classical 2D $X$-point reconnection. The faster the magnetic field expands with height, the slower and more complex are the dynamics of the magnetic reconnection at the early stages of its evolution.
\end{abstract}

Key words. MHD - Sun: atmosphere - Sun: transition region - Sun: magnetic fields

\section{Introduction}

The wealth and puzzle of the Sun provides a great variety of dynamic phenomena interpretable as manifestations of magnetic reconnection. These range from the classical small-scale explosive events that are observed on the "quiet" Sun, through to the violent flares produced in active regions. The high degree of complexity of the magnetic field inferred from observations may locally produce a fruitful environment for the occurrence of magnetic reconnection.

To date, there are extensive numerical studies of phenomena associated with active regions (such as solar flares, $X$-ray jets, etc.), involving fast magnetic reconnection (see Forbes \& Priest 1984; Yokoyama \& Shibata 1996; Chen et al. 1999). These models successfully predict most of the observational signatures of violent flare events found in recent X-ray observations by Yohkoh. It is also worth mentioning the pioneer numerical work on the subject by Ugai \& Tsuda (1977), in which the dynamics of magnetic

Send offprint requests to: I. Roussev, e-mail: ilr@ucar.edu reconnection driven by locally enhanced resistivity was studied in great detail. This study suggested that, regardless of the imposed boundary conditions, the global flow structure can spontaneously be set up as the magnetic field lines reconnect.

There is now sufficient observational evidence that solar explosive events are associated with regions undergoing magnetic reconnection. As originally pointed out by Dere et al. (1991), and later supported by Priest (1998), some of these events are most probably caused by magnetic flux cancellation between the pre-existing network magnetic field and newly emerging magnetic flux. It was found that there is a higher tendency for explosive events to occur near the network cell boundaries than at cell centres (Dere et al. 1991). In some recent studies (Innes et al. 1997; Wilhelm et al. 1998), it was suggested that the spatial and temporal evolution of observed line profiles during solar explosive events is consistent with physical interpretations involving bi-directional plasma flows. Observed Doppler shifts indicate bulk motions with velocities comparable to the local Alfvén speed (Dere et al. 1991). Thus, magnetic reconnection has become the strongest 
candidate capable of explaining the observational signatures of these events.

On the other hand, there are alternative physical mechanisms proposed to explain the nature of solar explosive events. It has been suggested that these are chromospheric evaporations as a result of coronal micro-flares (see Krucker et al. 1997; Wilhelm 2000). Also, the idea of swirling funnels of gas, i.e., the so-called solar tornadoes, can be used to explain the observed red- and blueDoppler shifts during explosive events (see Pike \& Mason 1998). In order to explore this phenomenon in depth, and thus give a firm answer about its true nature, higher spatial and temporal resolutions are required in the relevant observations.

There are some recent 2D MHD simulations related to explosive events (Roussev et al. 2001a,b,c). These magnetic reconnection model investigations also involve line synthesis of two resonance transition region lines, namely C IV $1548.2 \AA$ and O VI $1031.9 \AA$, considering non-equilibrium ionization. The effects of nonlinear fieldaligned thermal conduction, radiative losses, and volumetric heating are also taken into account. Furthermore, a detailed comparison was made between the dynamic responses of various physical conditions representing the "quiet" Sun to a magnetic reconnection event. It was found that the dynamical time-scales involved in the reconnection process strongly depends on the assumed arbitrary initial state.

Until now, there is a lack of studies of magnetic reconnection in a stratified physical environment. In this investigation, gravity is taken into account. We found that it has an influence that goes beyond just adding an additional external force term in the momentum equation. The presence of gravity also influences the structure of the initial magneto-hydrostatic solution. The initial state is determined in a more consistent way compared to the previously explored initial configurations. Note that all considerations here involve 2D MHD.

\section{Physical formulation of the problem}

In the following section, we present our 2D model. The governing equations of magnetohydrodynamics, including gravity, are given in Sect. 2.1. A description of the initial magneto-hydrostatic state is made in Sect. 2.2. Finally, in Sect. 2.3, we present the set of experiments that will be investigated.

\subsection{Basic equations}

The full set of equations of dissipative MHD, including gravity, reads

$\frac{\mathrm{D} \rho}{\mathrm{D} t}=-\rho \nabla \cdot \boldsymbol{U}$

$\rho \frac{\mathrm{D} \boldsymbol{U}}{\mathrm{D} t}=-\nabla p+\boldsymbol{j} \times \boldsymbol{B}+\rho \boldsymbol{g}-\nabla \cdot \hat{\tau}$ $\nabla \cdot \boldsymbol{B}=0$

$$
\frac{\mathrm{D} \boldsymbol{B}}{\mathrm{D} t}=\boldsymbol{B} \cdot \nabla \boldsymbol{U}-\boldsymbol{B} \nabla \cdot \boldsymbol{U}-\nabla \times\left[\left(\eta+\eta_{\mathrm{loc}}\right) \nabla \times \boldsymbol{B}\right],
$$

$\frac{\mathrm{D} e}{\mathrm{D} t}=-\gamma e \nabla \cdot \boldsymbol{U}+Q_{\mathrm{visc}}+Q_{\text {Joule }}$

Here $\mathrm{D} / \mathrm{D} t \equiv \partial / \partial t+\boldsymbol{U} \cdot \nabla$, and $\gamma, \rho, p, e, \boldsymbol{U}, \boldsymbol{B}, \boldsymbol{j}, \boldsymbol{g}$, $\eta, \hat{\tau}, Q_{\text {visc }}, Q_{\text {Joule }}$ are the adiabatic index, mass density, kinetic gas pressure, thermal energy, fluid velocity, magnetic field, electric current density, the field of gravity, magnetic diffusion coefficient ${ }^{1}$ (numerical), viscous stress tensor (numerical), viscous dissipation, and Joule dissipation, respectively. By $\eta_{\text {loc }}$ we denote a coefficient of localised ad hoc magnetic diffusion. This is needed in the experiments to break the initial magneto-hydrostatic equilibrium by igniting a localised magnetic reconnection process. In addition, $\boldsymbol{j}$ and $p$ are explicitly given by

$\boldsymbol{j}=\nabla \times \boldsymbol{B}, \quad p=e(\gamma-1)=\rho T$.

Note here that we are working in units where the gas constant equals the mean molecular weight.

\subsection{Initial states}

In our previous investigations (Roussev et al. 2001a,b), various initial states were constructed without including gravitational stratification. In this study, gravity is taken into account because it has a non-trivial role in defining the initial state. This is achieved in a $2 \mathrm{D}$ case where a unique solution has been found.

Let us denote by $L_{0}, V_{\mathrm{A} 0}, t_{\mathrm{A} 0}, \rho_{0}, T_{0}, p_{0}, e_{0}, B_{0}$, and $j_{0}$, a typical length scale, velocity, time scale, mass density, temperature, kinetic gas pressure, thermal energy, magnetic field strength, and electric current density, respectively. In the present investigation, all of these parameters are chosen to represent the "quiet" solar transition region environment. Assigned values for $L_{0}, V_{\mathrm{A} 0}, t_{\mathrm{A} 0}, \rho_{0}, T_{0}$, and $B_{0}$ are given in Table 1 (in CGS units). As to $V_{\mathrm{A} 0}, t_{\mathrm{A} 0}$, $p_{0}, e_{0}$, and $j_{0}$, the following formulae apply

$V_{\mathrm{A} 0}=\frac{B_{0}}{\sqrt{\rho_{0}}}, t_{\mathrm{A} 0}=\frac{L_{0}}{V_{\mathrm{A} 0}}, p_{0}=\beta_{0} \frac{B_{0}^{2}}{2}, e_{0}=\frac{p_{0}}{\gamma-1}$,

and

$j_{0}=\frac{B_{0}}{L_{0}}$

where $\beta_{0}$ is the plasma "beta" parameter at some reference location (to be explained below) in the domain.

Let us now define dimensionless physical quantities, that are denoted by, $t^{*}, \boldsymbol{u}, \boldsymbol{b}, \boldsymbol{g}^{*}, \rho^{*}, T^{*}, p^{*}, e^{*}$, and $\boldsymbol{j}^{*}$.

1 We work in units where the magnetic permeability is set to 1 , and thus the resistivity and magnetic diffusivity are the same from a numerical point of view. 
The transformation relations between the real physical quantities and these normalised variables (which are adopted in the numerical code) are given by

$t^{*}=\frac{t}{t_{\mathrm{A} 0}}, \boldsymbol{u}=\frac{\boldsymbol{U}}{V_{\mathrm{A} 0}}, \boldsymbol{b}=\frac{\boldsymbol{B}}{B_{0}}, \boldsymbol{g}^{*}=\boldsymbol{g} \frac{t_{\mathrm{A} 0}}{V_{\mathrm{A} 0}}$,

$\rho^{*}=\frac{\rho}{\rho_{0}}, T^{*}=\frac{T}{T_{0}}, p^{*}=\frac{p}{p_{0}}, e^{*}=\frac{e}{e_{0}}$,

and

$j^{*}=\frac{j}{j_{0}}$

In addition, we introduce the normalised coordinates $x^{*}$ and $y^{*}$

$x^{*}=\frac{x}{L_{0}}, y^{*}=\frac{y}{L_{0}}$,

where $x_{\min }^{*} \leq x^{*} \leq x_{\max }^{*}, y_{\min }^{*} \leq y^{*} \leq y_{\max }^{*}$, and $x_{\min }^{*}=$ $-x_{\max }^{*}$ (similarly, $y_{\min }^{*}=-y_{\max }^{*}$ ). The normalisation units that are given in Table 1 refer to the particular location with coordinates $\left(x_{\min }^{*}, y_{\min }^{*}\right)$. All derivations hereafter are made for the normalised physical quantities.

The optimal situation would be to fix the parameters representing the diffusion region, and thereby make the physical environment identical for each experiment. By doing this, the initial conditions would be restricted to a small parameter regime, which depends on the actual size of the numerical domain. These restrictions reflect the physical requirement that both the plasma density and kinetic gas pressure (defined below) must be positive inside the numerical domain. By using the particular point, $\left(x_{\min }^{*}, y_{\min }^{*}\right)$, this requirement can be fulfilled for a much larger parameter regime, but with an effect that the physical parameters in the diffusion region change.

In the 2D models, gravity is in the negative $y$-direction, with a normalised, constant acceleration, $g^{*}$, given by $g^{*}=g_{0} t_{\mathrm{A} 0} / V_{\mathrm{A} 0}$. The assigned value for $g_{0}$ is given in Table 1 .

In the initial state, the plasma is considered to be at rest, i.e. $\boldsymbol{u}=0$. The initial magnetic field, $\boldsymbol{b}=\left(b_{x}, b_{y}\right)$, is then prescribed by

$b_{x}\left(x^{*}, y^{*}\right)=\frac{m}{b c} \ln \left[\cosh \left(b x^{*}\right)\right] \exp \left(-\frac{y^{*}}{c}\right)$,

and

$b_{y}\left(x^{*}, y^{*}\right)=m \tanh \left(b x^{*}\right) \exp \left(-\frac{y^{*}}{c}\right)$,

where the constant amplitude $m$ is chosen such that the magnetic field is unity at the reference location $\left(x_{\min }^{*}, y_{\min }^{*}\right)$. The variables $c$ and $b$ are two free model parameters, where $c$ defines the scale-height of the magnetic field, and $b$ controls the width of the current concentration around the symmetry axis $x^{*}=0$. The magnetic field described by Eqs. (7), (8) represents a situation where two magnetic flux tubes of opposite polarity have a contact at $x^{*}=0$.
Table 1. Normalisation units.

\begin{tabular}{|lll|}
\hline Physical quantity & Notation & Typical value \\
\hline \hline Length & $L_{0}$ & $1.5 \times 10^{8}(\mathrm{~cm})$ \\
Velocity & $V_{\mathrm{A} 0}$ & $1.293 \times 10^{7}\left(\mathrm{~cm} \mathrm{~s}^{-1}\right)$ \\
Time & $t_{\mathrm{A} 0}$ & $1.16 \times 10^{1}(\mathrm{~s})$ \\
Gravity & $g_{0}$ & $2.7 \times 10^{4}\left(\mathrm{~cm} \mathrm{~s}^{-2}\right)$ \\
Density & $\rho_{0}$ & $5.02 \times 10^{-14}\left(\mathrm{~g} \mathrm{~cm}^{-3}\right)$ \\
Temperature & $T_{0}$ & $1 \times 10^{5}(\mathrm{~K})$ \\
Magnetic field & $B_{0}$ & $8(\mathrm{G})$ \\
\hline
\end{tabular}

Since the initial state is taken to be in static equilibrium, the Lorentz and gravity forces have to be balanced by the pressure gradient in the entire physical domain. This requirement is determined by the static momentum equation

$\frac{\partial p^{*}}{\partial x^{*}}+j^{*} b_{y}=0$

and

$\frac{\partial p^{*}}{\partial y^{*}}-j^{*} b_{x}+\rho^{*} g^{*}=0$.

Here $j^{*}$ is the only non-zero component of the electric current density given by $j^{*}=\left(\nabla^{*} \times \boldsymbol{b}\right)_{z}$, where $\nabla^{*}=$ $L_{0} \nabla$. These equations can be solved in order to obtain the mass density, $\rho^{*}$, and kinetic gas pressure, $p^{*}$, using the expressions for the magnetic field given by Eqs. (7), (8). Corresponding solutions are

$$
\begin{aligned}
& \rho^{*}=\left\{\frac{m^{2}}{c g^{*}}\{1+\ln \right. {\left.\left.\left[\cosh \left(b x^{*}\right)\right]\right\} \operatorname{sech}^{2}\left(b x^{*}\right)+\rho_{0}^{*}\right\} } \\
& \times \exp \left(-2 \frac{y^{*}}{c}\right)
\end{aligned}
$$

$$
\begin{gathered}
p^{*}=\left\{\frac{m^{2}}{2 b^{2} c^{2}}\left\{b^{2} c^{2} \operatorname{sech}^{2}\left(b x^{*}\right)-\ln ^{2}\left[\cosh \left(b x^{*}\right)\right]\right\}\right. \\
\left.+\frac{\rho_{0}^{*} g^{*} c}{2}\right\} \exp \left(-2 \frac{y^{*}}{c}\right)+p_{0}^{*}
\end{gathered}
$$

where $\rho_{0}^{*}$ and $p_{0}^{*}$ are two integration constants chosen such that $\rho^{*}\left(x_{\text {min }}^{*}, y_{\text {min }}^{*}\right)=1$, and $p^{*}\left(x_{\text {min }}^{*}, y_{\text {min }}^{*}\right)=\beta_{0} / 2$. Here $\beta_{0}$ is the plasma 'beta' parameter at the reference location.

The corresponding plasma temperature, $T^{*}$, is determined by $T^{*}=p^{*} / \rho^{*}$, and therefore at the reference location $T^{*}\left(x_{\text {min }}^{*}, y_{\text {min }}^{*}\right)=\beta_{0} / 2$. In this $2 \mathrm{D}$ MHD model of a stratified atmosphere described by Eqs. (7)-(12), there are fewer free parameters than in the case without gravity (see Roussev et al. 2001a). Once the choice regarding $L_{0}$ and $t_{\mathrm{A} 0}$ is made, the value of $g^{*}$ is automatically defined, $g^{*}=g_{0} t_{\mathrm{A} 0} / V_{\mathrm{A} 0}$. The freedom in the initial configuration is then given with regard to the choice of the $\beta_{0}, b, c$, and $m$ parameters. 
The initial state has an Alfvén velocity that is uniform with height, and increases with the horizontal distance from the central current sheet. In contrast to this, the plasma "beta" parameter depends on both spatial coordinates, and is found to increase with height. The actual dependence is controlled by the value of the scale-height, $c$. The smaller $c$ is, the faster $\beta$ increases with height. Once the scale-height is set to infinity, $\beta$ becomes uniform with height. Furthermore, in the limit case of $c=\infty$ and $g^{*}=0$, we obtain the initial configuration used in experiment "B2" of Roussev et al. (2001b). This represents a physical situation of a hot current "sheet" surrounded by cold plasma, where the temperature is constant with height and the mass density is uniform in the entire physical domain.

The increase of plasma $\beta$ with height is not what we expect in the real solar atmosphere. The above initial state has this unrealistic feature because it is a $2 \mathrm{D}$ model. In a $2.5 \mathrm{D}$ model, however, this unwanted feature could easily be avoided by adding a strong enough constant magnetic field in direction out of the $2 \mathrm{D}$ plane. In this case, all derivations made above still apply. The only difference appears in the $y$-dependence of $\beta$. Such an extension to $2.5(3) \mathrm{D}$ model would therefore be a more realistic representation of a stratified solar atmosphere.

\subsection{Set of experiments}

In the present study, we explore the dynamical evolution of magnetic reconnection in various physical conditions representing a 2D stratified solar atmosphere. Based on the initial setup presented in the previous section, Eqs. (7)-(12), five different initial states are constructed. These differ from each other by the assigned value for the scale-height, $c$, and the magnetic field strength, $m$. Note that the $m$ parameter mostly depends on the scale-height through $\exp \left(y_{\min }^{*} / c\right)$ and therefore scales with the scaleheight, approaching 1 as $c \rightarrow \infty$ (if $y_{\min }^{*}<0$ ). The exact parameters chosen for each of the five experiments are given in Table 2.

In the initial state, the most rapid decline with height is found in experiment "G1", while the longest scale-height is in experiment "G5". These are the two extreme cases that will be discussed in the next section. As mentioned earlier, these parameter choices make the physical conditions around the inflow regions of the reconnection site slightly different. This is seen in Table 3 which shows the plasma $\beta$ in the inflow regions for the five experiments, $\beta_{i}$. This parameter is defined at $\left(x^{*}, y^{*}\right)=\left(x_{\min }^{*},-y_{0}^{*}\right)$, where $-y_{0}^{*}$ is the height (measured from the center of the domain) at which the reconnection process is initiated. The influence of the change in plasma $\beta$ is investigated through a couple of experiments using the "G1" setup and changing the plasma $\beta$ value by a factor of two and four, respectively. These experiments showed only small changes in the physical parameters of the jets, indicating that moderate changes in the plasma $\beta$ have only minor influence on the dynamics of the reconnection process compared to the influence of the stratification.

Similar to our previous studies (refer to Roussev et al. 2001a,b), magnetic reconnection is driven in an ad hoc manner. This is achieved by switching on a localised magnetic diffusion, $\eta_{\mathrm{loc}}^{*}$, in the $2 \mathrm{D}$ current concentration where the magnetic field reverses polarity. The spatial form and time-dependence of the ad hoc diffusivity are given by

$$
\begin{aligned}
\eta_{\mathrm{loc}}^{*}\left(x^{*}, y^{*} ; t^{*}\right)= & \eta_{0}^{*} F^{*}\left(t^{*}\right) \\
& \times \exp \left\{-\alpha\left[x^{* 2}+\left(y^{*}+y_{0}^{*}\right)^{2}\right]\right\},
\end{aligned}
$$

where

$$
F^{*}\left(t^{*}\right)= \begin{cases}\frac{t^{*}}{t_{\mathrm{ri}}^{*}}, & 0 \leq t^{*} \leq t_{\mathrm{ri}}^{*} \\ 1 ; & t_{\mathrm{ri}}^{*}<t^{*} \leq t_{\mathrm{off}}^{*} \\ 0 ; & t_{\mathrm{off}}^{*}<t^{*}\end{cases}
$$

Here $\eta_{0}^{*}$ is the normalised value of the localised magnetic diffusion coefficient. The spatial shape of $\eta_{\mathrm{loc}}^{*}$ is a double Gaussian with a half-width controlled by the $\alpha$ parameter. The driver term given by Eq. (14), increases linearly from time $t^{*}=0$ until $t_{\mathrm{ri}}^{*}$, followed by a constant value up to $t^{*}=t_{\mathrm{off}}^{*}$. At time $t_{\mathrm{off}}^{*}$, the driver term is turned off. Assigned values for all these parameters are given in Table 2. Notice that the centre of the diffusion region is moved down relative to the centre of the domain by $y_{0}^{*}$. This is done in order to allow the generally faster upward propagating jet a longer distance to travel before the experiment has to be terminated. In all experiments, the ad hoc diffusion coefficient is switched on all the time in order to have a constant localised "driving" of the reconnection process.

The seven experiments are carried out by using a 2D compressible MHD code based on staggered meshes (for more details refer to Nordlund \& Galsgaard 1995; Roussev et al. 2001a). The MHD equations are solved in a 2D domain, with a stretching applied with respect to the $x$-coordinate. This is done in order to achieve a high numerical resolution in the current concentration, where the most important dynamics takes place, and to move the $x$-boundaries as far away as possible with a minimum number of grid-points. Throughout the experiments, the velocities at the boundaries are maintained at zero. The experiments are therefore terminated as the reconnection jets approach the boundaries.

The numerical grid-size is $N_{x} \times N_{y}=601 \times 801$. The computational domain is chosen to be of size $\left[2 x_{\max }^{*}, 2 y_{\max }^{*}\right]=[6,10]$. Stretching of the grid in the $x$ direction is applied in such a way that $50 \%$ of the grid points are located within $-0.7 \leq x^{*} \leq 0.7$. The smallest grid spacing is assigned across the symmetry axis $x^{*}=0$, and corresponds to $\left(\Delta x^{*}\right)_{\min }=3.96 \times 10^{-3}$. At the $x$-boundaries, the stretching gives $\left(\Delta x^{*}\right)_{\max }=$ $4.40 \times 10^{-2}$. For comparison, the grid spacing in the $y$-direction is uniform and $\Delta y^{*}=1.25 \times 10^{-2}$. 
Table 2. Parameters for the various experiments.

\begin{tabular}{|llllll|}
\hline Exp. & G1 & G2 & G3 & G4 & G5 \\
\hline \hline$\gamma$ & $5 / 3$ & $5 / 3$ & $5 / 3$ & $5 / 3$ & $5 / 3$ \\
$\beta_{0}$ & 0.164 & 0.164 & 0.164 & 0.164 & 0.164 \\
$g^{*}$ & $2.42 \times 10^{-2}$ & $2.42 \times 10^{-2}$ & $2.42 \times 10^{-2}$ & $2.42 \times 10^{-2}$ & $2.42 \times 10^{-2}$ \\
$b$ & 25 & 25 & 25 & 25 & 25 \\
$c$ & $\mathbf{5 / 3}$ & $\mathbf{1 0} / \mathbf{3}$ & $\mathbf{5}$ & $\mathbf{1 5}$ & $\mathbf{4 5}$ \\
$m$ & $\mathbf{0 . 0 2 4}$ & $\mathbf{0 . 1 6 7}$ & $\mathbf{0 . 3 1 6}$ & $\mathbf{0 . 7 0 3}$ & $\mathbf{0 . 8 9 3}$ \\
$\eta_{0}^{*}$ & $1 / 200$ & $1 / 200$ & $1 / 200$ & $1 / 200$ & $1 / 200$ \\
$\alpha$ & 150 & 150 & 150 & 150 & 150 \\
$t_{\mathrm{ri}}^{*}$ & $5 / 100$ & $5 / 100$ & $5 / 100$ & $5 / 100$ & $5 / 100$ \\
$t_{\mathrm{off}}^{*}$ & 5 & 5 & 5 & 5 & 5 \\
$y_{0}^{*}$ & $5 / 10$ & $5 / 10$ & $5 / 10$ & $5 / 10$ & $5 / 10$ \\
\hline
\end{tabular}

Table 3. Plasma $\beta$ values for the inflow regions.

\begin{tabular}{|llllll|}
\hline Exp. & G1 & G2 & G3 & G4 & G5 \\
\hline \hline$\beta_{i}$ & 0.861 & 0.362 & 0.276 & 0.196 & 0.174 \\
\hline
\end{tabular}

\section{Discussion of the numerical results}

Here we discuss the numerical results obtained from the present modelling. The general dynamics of magnetic reconnection in the experiments is analysed in Sect. 3.1. The deviations from traditional $2 \mathrm{D} X$-point reconnection discovered at the early phases of this process are discussed in Sect. 3.2. A further comparison between the five experiments is made in Sect. 3.3.

\subsection{Dynamics of the reconnection process}

The 2D current concentration in the initial background plasma begins to dissipate when the ad hoc localised magnetic diffusion, Eq. (14) is turned on at time $t^{*}=0$. This forces the magnetic field in the diffusion region to restructure. In all of the models examined here, the localised diffusivity is induced in the same spatial and temporal manner, and is kept active until $t_{\text {off }}^{*}=5$. The numerical experiments are then followed until the reconnection driven jets reach the boundaries of the computational domain. This occurs at $t_{\text {end }}^{* \mathrm{G} 1}=7.00$ (for experiment "G1"), $t_{\text {end }}^{* \mathrm{G} 2}=6.50$ (for experiment "G2"), $t_{\text {end }}^{* \mathrm{G} 3}=5.85$ (for experiment "G3"), $t_{\mathrm{end}}^{* \mathrm{G} 4}=4.60$ (for experiment "G4"), and $t_{\text {end }}^{* \mathrm{G} 5}=4.40$ (for experiment "G5"), respectively. In the following analysis, however, we are only interested in the time-evolution of the MHD solutions while the ad hoc diffusivity is active, and thus limit our discussion to times less than $t^{*}=5$.

As the localised diffusivity starts changing the magnetic field structure, the initial pressure balance is destroyed. The diffusion of magnetic field generates new reconnected field lines. These possess high tension forces within the initial current concentration. The plasma in the diffusion region is pulled out by these field lines, and accelerated in $\pm y$-directions by the strong tension forces.

Since the magnetic field strength declines with height, and as this field spreads out in the $\pm x$-directions around the current concentration, the resulting Lorentz forces of newly reconnected field lines have different magnitudes on either side of the diffusion region along $y$. The downward directed Lorentz force is larger than the one directed upwards. Furthermore, the effect of gravity and the change in mass density with height add to the asymmetry of the resulting outflow velocities of the two reconnection driven jets. The faster the initial magnetic field decreases with height, i.e. the smaller the value of the $c$ parameter, the larger is the difference between the magnetic tension forces in the opposite $y$-directions. Thus, one would expect the largest differences in the dynamics of the jets to be found between experiments "G1" $(c=5 / 3)$ and "G5" $(c=45)$.

Once magnetic reconnection starts, both the kinetic gas and magnetic pressures decrease in the region of localised magnetic diffusion. This initiates a pressure driven inflow of magnetised plasma towards the diffusion region from outside the current concentration. This plasma flow advects new magnetic flux into the diffusion region, and thus a continuous reconnection process is sustained. As a result, two outflow jets, the so-called reconnection jets, are naturally formed. As these jets dynamically expand, the non-heated plasma surrounding the current concentration is pushed away. The jet motions around the current structure subsequently steepen into bow shocks. These are fast-mode shocks since the magnetic field strength in the downstream region is increased. The thermal energy inside the reconnection jets increase with time as a result of kinetic energy being converted into heat through shocks, and also due to the nearly adiabatic plasma compression. Hence, the outflow jets dynamically expand in the $x$-directions as they propagate along $\pm y$. The faster the magnetic field strength decreases with height, the 

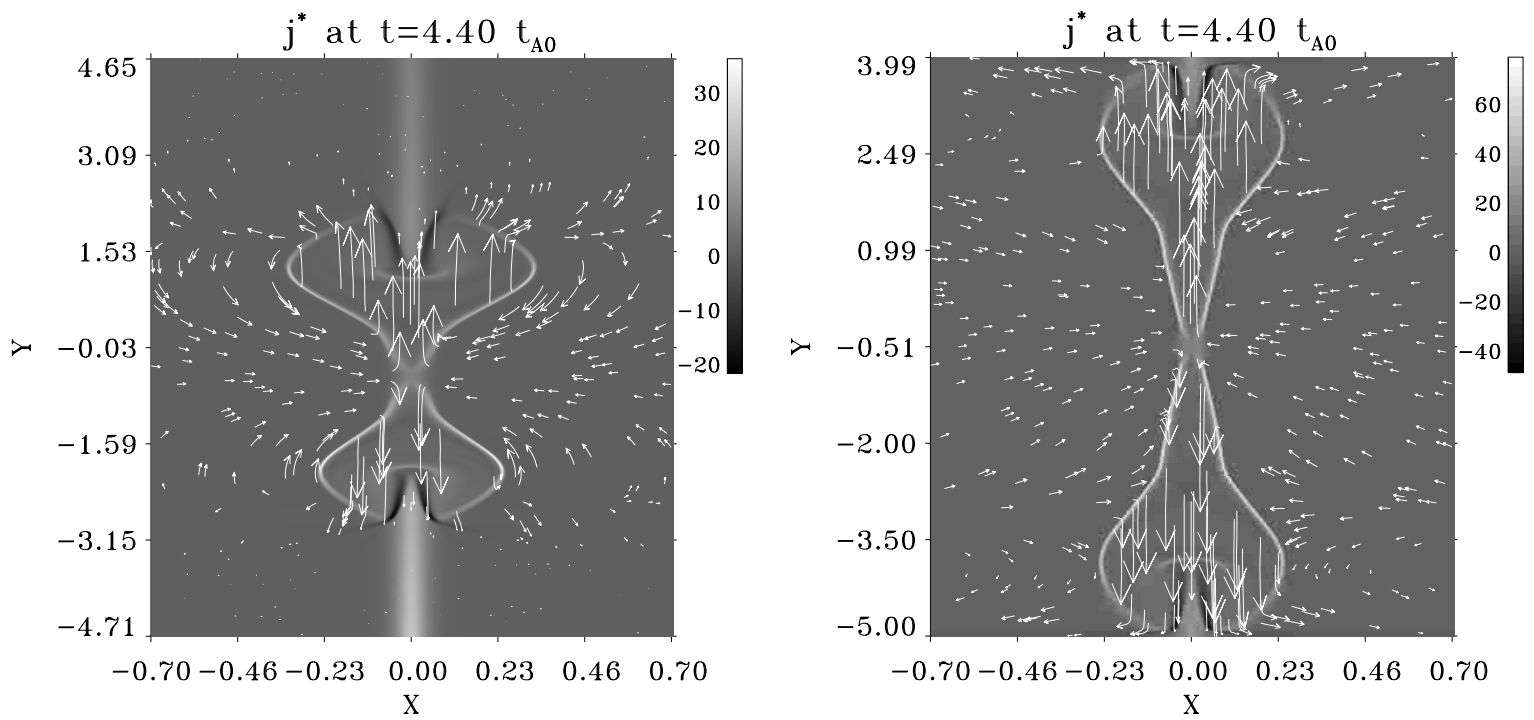

Fig. 1. The current density and velocity field structure are shown for experiments "G1" (left panel) and "G5" at time $t^{*}=4.4$. The former quantity is visualised as a grey-scale background image, whose scaling is indicated on the adjacent grey-scale bar. Short streak lines are traced from random starting positions in order to represent the velocity field pattern at this time.
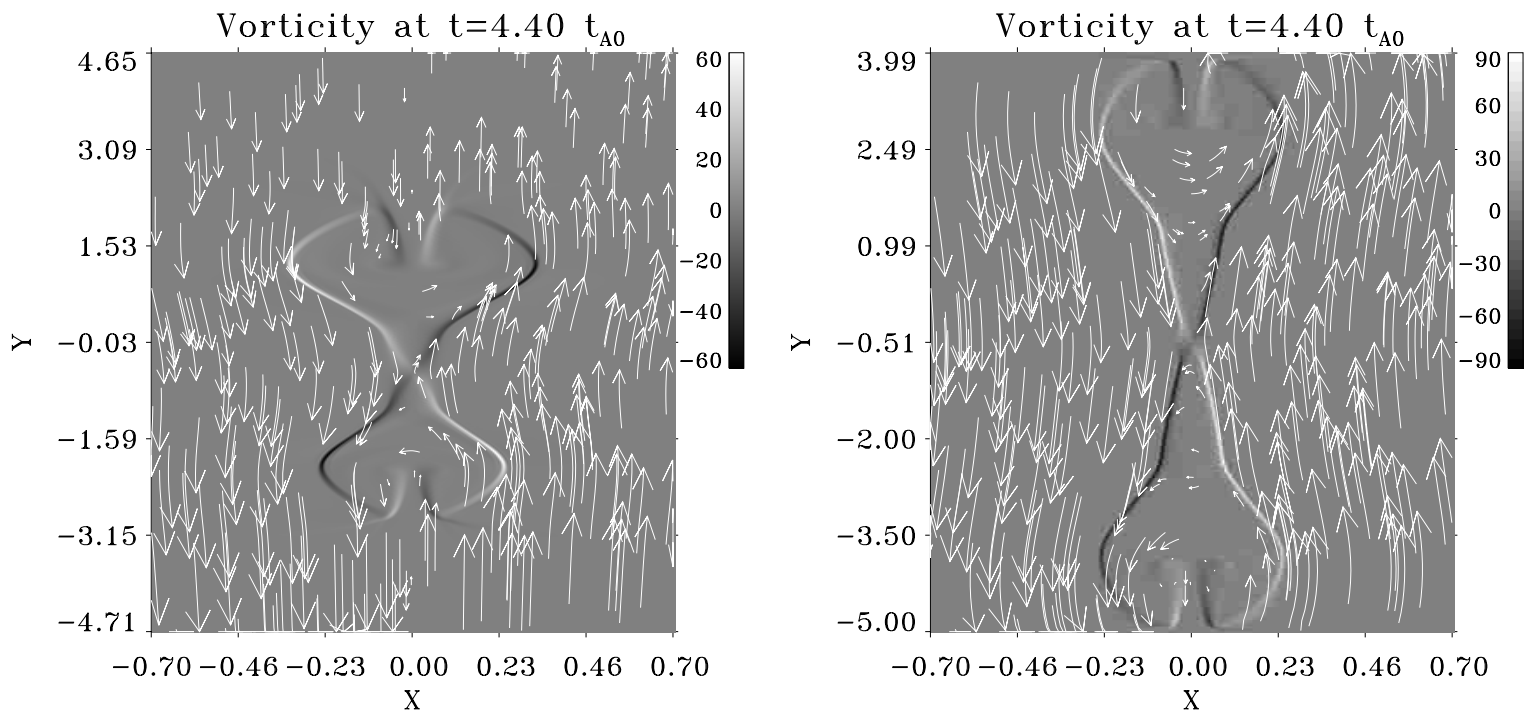

Fig. 2. The vorticity and magnetic field line structure are shown for experiments "G1" (left panel) and "G5" at the same time as in Fig. 1. The vorticity is shown as a grey-scale background image, whose scaling is indicated on the attached grey-scale bar. Short field line segments are traced from random start positions to indicate the magnetic field topology at this time.

farther the reconnection jets expand in the horizontal direction. The larger the expansion of the jets are, the smaller the pressure difference between the inside and the outside of the jets. Their confined structure is provided by the Lorentz force of the ambient magnetic field in the form of standing slow-mode shocks. The shocks are classified as such because the magnetic field strength in the downstream region is decreased. The enhanced current density in the slow-mode shocks scales inversely with the expansion of magnetic field with height. Thus, at a given height, the smaller the scale-height of the model, the larger the jet expansion in the horizontal direction is expected to be. All this is confirmed by the results shown in Fig. 1 . This figure shows the current density at time $t^{*}=4.4$, for experiments "G1" and "G5" (right panel). In addition, short streak lines are traced from random starting positions in order to visualise the velocity field at this time. The standing slow-mode shocks are recognised as locations of strong, positive current concentrations (bright lanes). These occur because of the steep gradient of the parallel magnetic field component across the shocks, with the after-shock field strength being decreased. The bow shocks are recognised in this panels as regions of strong, negative current density (dark lanes).

In 2D magnetic reconnection, another good indicator of the locations of slow-mode shocks are the separatrix lines which divide the inflow from the outflow regions formed by the reconnection process. The separatrices can be traced by the locations of large vorticity, as seen in Fig. 2. This figure shows the vorticity and associated 

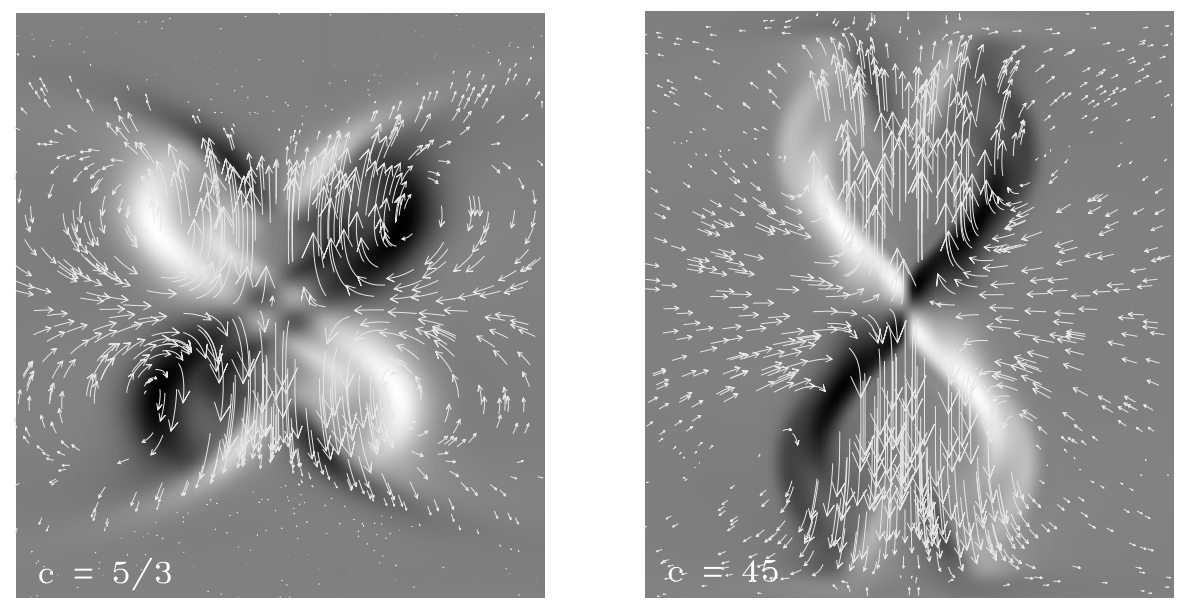

Fig. 3. Images of the vorticity are shown for experiments "G1" and "G5" (right panel) at time $t^{*}=0.85$. Short streak lines are plotted to represent the actual velocity field at this time. The size of the plotted region represents $[-0.18,0.18]$ in the $x^{*}$-direction and $[-1.4,0.4]$ in the $y^{*}$-direction.

magnetic field topology for experiments "G1" and "G5" (right panel) at the same time as in Fig. 1. Here the vorticity is visualised as the grey-scale shaded background image, and the magnetic field structure is indicated by tracing segments of field lines with randomly chosen starting points. The locations of slow-mode shocks are seen as thin black and white lines in vorticity. By comparing the plots of the two experiments, it is obvious that the difference in magnetic structure determined by the scale-height has important implications for the evolution of the reconnection event. For large scale-heights, the magnetic field structure is nearly independent of height, and the two outflow jets appear almost identical. When the scale-height is decreased, the magnetic field expands away from the current "sheet" and its strength decreases with height. The left panels in both figures show how this gives rise to the expected asymmetry between the two jets, with the upward propagating jet expanding much more in the horizontal direction than the jet moving downwards. Furthermore, as the two panels in Fig. 1 represent the same physical time, it is seen that the velocity of the jet scales with the scale-height.

If observations of the physical extent of reconnection jets in the solar atmosphere were possible, then this could be used, through more advanced models, to provide information about the fine structure of the magnetic field with height. This could be a possible task for future high resolution observations.

As it was already predicted from the discussion above, the strength of the slow-mode shocks scales with the scaleheight. This is clearly seen from the dynamical range covered by the shading of vorticity, as indicated by the grey-scale bar attached to each panel in Fig. 2. At a well developed stage of the reconnection process, the traditional quadrupolar structure of the vorticity is followed (Priest \& Forbes 1986).

Further discussions of the jet dynamics in the various experiments are given in Sect. 3.3 below.

\subsection{Early phases of the reconnection process}

In both panels of Fig. 2, we recognise the classical scheme of 2D $X$-point magnetic reconnection with a distinct quadrupolar structure of the vorticity in vicinity of the diffusion region. This is, however, not the case in the early stages of the reconnection events. A close-up at the vorticity at time $t^{*}=0.85$ shows a different regime of magnetic reconnection in experiment "G1", as seen in the left panel of Fig. 3. Here the right panel refers to experiment "G5", and in contrast, shows the traditional regime $2 \mathrm{D} X$-point reconnection. The background shading in both panels represents the vorticity visualised as a grey-scale image, while the short streak lines represent the actual velocity field at this time. Note also that both panels show the same spatial regions.

As seen in Fig. 3, the vorticity in experiment "G1" reveals a rather complex signature. The vorticity pattern is found to contain a nested sequence of quadrupolarlike structures ("chess-mate" pattern). The lifetime of this phase is short, and it subsequently evolves into the traditional 2D $X$-point reconnection scheme, characterised with only one vorticity quadrupole. The transition time between the two regimes is found to scale inversely to the scale-height, $c$.

By investigating the changes in the vorticity pattern for all five experiments, the duration of this phase is found to be $t_{\mathrm{tr}}^{* \mathrm{G} 5}=0.70$ for experiment "G5", while in experiment "G1" that lasts until $t_{\mathrm{tr}}^{* \mathrm{G} 1}=1.75$. In the three intermediate experiments, the transition occurs at times $t_{\mathrm{tr}}^{* \mathrm{G} 2}=1.15$ for experiment "G2", $t_{\mathrm{tr}}^{* \mathrm{G} 3}=0.95$ for experiment "G3", and $t_{\mathrm{tr}}^{* \mathrm{G} 4}=0.75$ for experiment "G4", respectively.

The complex vorticity signature at early phases of the reconnection process is caused by initial deviations from the total force balance. This occurs since the magnetic field diffuses away faster than the pressure gradient reacts to the changes in the Lorentz force (i.e., the 
magnetic Reynolds number is less than unity). As a result, the Lorentz force gets a double-wave pattern in the horizontal direction. This combined with the very initial compressing effect of the Lorentz force in the vertical direction produces a complex vorticity pattern, like the one shown in the left panel of Fig. 3. The alternating signs of vorticity follow those of the Lorentz forces with respect to the $x^{*}$ and $y^{*}$ coordinates. This initial phase can only survive as long as the diffusive part of the induction equation dominates the dynamics of the diffusion region. When the advection of material from outside this region becomes large enough, the complex vorticity pattern is suppressed and eventually vanishes. This occurs when the advective term in the induction equation becomes comparable in magnitude to the diffusive term, i.e., when the magnetic Reynolds number becomes larger than unity.

Similar results in a $1 \mathrm{D}$ current sheet were reported by Forbes et al. (1982). It was found that, if the speed for magnetic diffusion, controlled by the initial width of the current sheet, is greater than the sound speed, then isomagnetic shocks are formed. These shocks propagate outwards from the centre of the locally enhanced resistivity, and transform into fast magneto-acoustic waves as they approach the edges of the current sheet.

Although it is not clear from Fig. 3, the maximum jet velocity is reached along the symmetry axis, $x^{*}=0$, in all of the experiments. In the initial evolution, the profile of velocity magnitude across the current concentration in experiment "G1" is more shallow in the vicinity of $x^{*}=0$, than that in experiment "G5". In Fig. 3, the maximum flow velocity is 0.21 for experiment "G1", and 0.54 for experiment "G5", respectively. It therefore seems that the initial regime of magnetic reconnection with the complex vorticity pattern is less efficient in forming a well collimated outflow jet than in the traditional 2D $X$-point reconnection.

\subsection{Further comparison between the various experiments}

The initial temperature profile across the current concentration changes considerably as the scale-height is varied. At small scale-heights, an initial state with a cold and dense current concentration surrounded by hot plasma is obtained. In this case, the temperature gradient along the current concentration is smaller than that of the ambient regions. As the scale-height is increased, the initial temperature of the current concentration increases. In turn, as the scale-height approaches infinity, a hot 2D current concentration placed in cold surrounding plasma is obtained.

In Fig. 4, the relative changes in temperature, ( $T-$ $\left.T_{\text {in }}\right) / T_{\text {in }}$, are shown relative to the initial temperature, $T_{\mathrm{in}}$, for experiments "G1" and "G5" (right panel) at time $t^{*}=4.4$. The adjacent image to the right on each frame shows the decimal logarithm of the normalised temperature, $\log _{10}\left(T / T_{0}\right)$. The surface and contour plots are out of spatial scale, while the adjacent images show the correct aspect ratio between the $x^{*}$ and $y^{*}$-coordinates.

For further convenience, let us use the term "blue" jet for the one propagating in the positive $y$-direction, while "red" jet is used for the one moving in the opposite direction.

By comparing the two frames in Fig. 4, it can be seen that the initial current concentration in experiment "G1" (left) is cold relative to the one in experiment "G5" (right). As the reconnection process proceeds, the temperature in the part of current concentration affected by the reconnection jets is changed. As these jets advect thermal energy, affected regions experience a temperature increase due to both the nearly adiabatic compression of the jet plasma and the localised shock heating. Despite this general trend, two large areas of the "blue" jet in experiment "G1" experience a decrease in temperature relative to the initial one. This is due to the large jet expansion in the horizontal direction which results in an adiabatic cooling of the plasma.

The changes in mass density, $\left(\rho-\rho_{\text {in }}\right) / \rho_{\text {in }}$, relative to the initial density distribution, $\rho_{\text {in }}$, are shown in Fig. 5. The adjacent image to the right of each frame shows the decimal logarithm of normalised mass density, $\log _{10}\left(\rho / \rho_{0}\right)$. By comparing this figure with that of the temperature, one can see that both $\rho^{*}$ and $T^{*}$ have enhanced values within the regions confined by the jets, while their peak values are located at slightly different spatial locations. The thermal energy distribution inside the jets is rather uniform. This is why the spatial variations in mass density are seen in contrast to those of the plasma temperature. As for the temperature, there are structural differences between the two experiments due to the different initial conditions. There is also a significant difference in the relative enhancement of mass density in the two experiments, with the peak value scaling inversely with the scale-height.

In the following, we make a comparison between all five experiments with regard to the maximum flow velocity, $V_{\text {jet }}^{\mathrm{max}}$, reached in the reconnection jets. We also derived the mass density, $\rho_{\text {jet }}^{*}$ and temperature, $T_{\text {jet }}^{*}$, at the location of $V_{\text {jet }}^{\max }$. Our discussion is limited to the "blue" jets only. Similar qualitative results are obtained for the "red" jets.

The top left panel in Fig. 6 shows the peak flow velocities of the "blue" jets in all experiments as functions of time. The top right panel shows the peak jet velocity versus its $y^{*}$-location where time is measured along the curve starting from the origin at $y^{*}=-0.5$. The mass density (bottom left) and temperature (bottom right) at the location of maximum jet velocity are also derived and shown as functions of time.

The maximum jet velocity in all experiments increases with time and saturates around a certain value towards the end of the experiments, except for "G1" which has not had sufficient time to reach the peak jet velocity. This value is determined by the Alfvén speed of the plasma outside the initial current concentration. In Fig. 6, it is seen that the peak velocity at a given time increases 

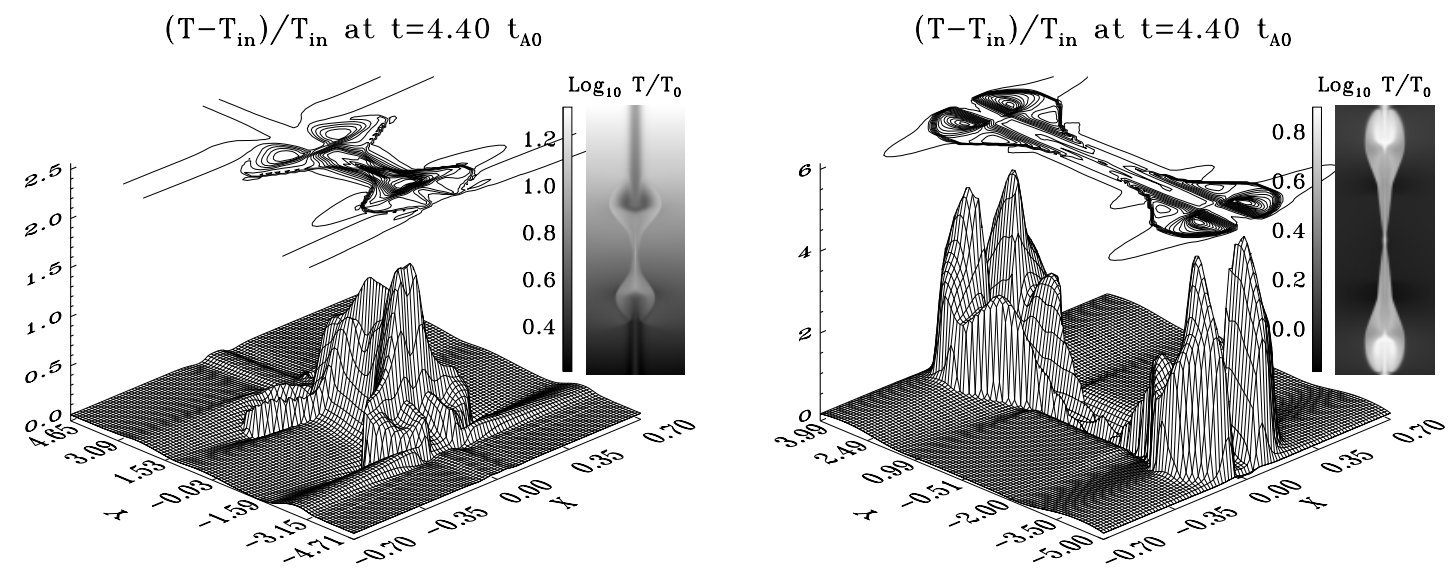

Fig. 4. Relative changes in plasma temperature, $\left(T-T_{\mathrm{in}}\right) / T_{\mathrm{in}}$, with respect to the initial temperature field, $T_{\mathrm{in}}$, are shown for experiments "G1" (left panel) and "G5" at time $t^{*}=4.4$. The adjacent image to the right in each panel shows the decimal logarithm of normalised temperature, $\log _{10}\left(T / T_{0}\right)$, at this time. Note that $T_{0}$ is the normalisation value of temperature, while $T_{\text {in }}$ is the initial temperature field in the physical domain.
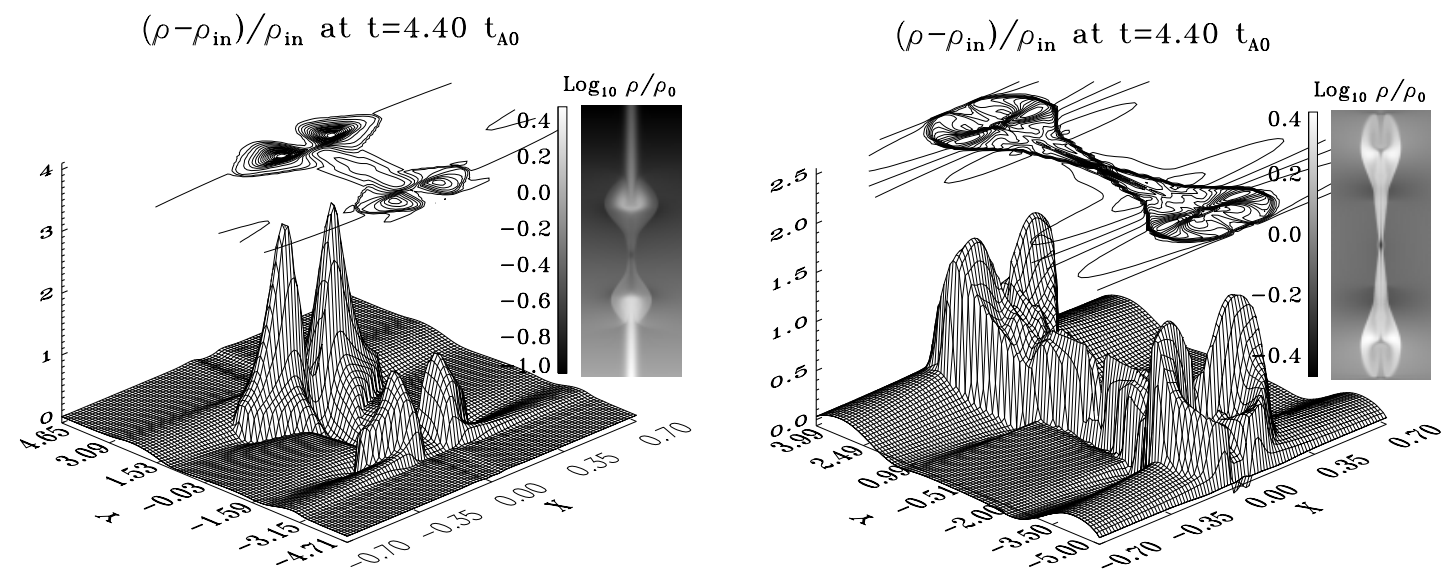

Fig. 5. Relative changes in mass density, $\left(\rho-\rho_{\text {in }}\right) / \rho_{\text {in }}$, with respect to the initial density distribution, $\rho_{\text {in }}$, are shown for experiments "G1" (left panel) and "G5" at the same time as in Fig. 4. The adjacent image to the right in each panel shows the decimal logarithm of normalised mass density, $\log _{10}\left(\rho / \rho_{0}\right)$, at this time. Note that $\rho_{0}$ is the normalisation value of mass density, while $\rho_{\text {in }}$ is the initial mass density distribution in the physical domain.

non-linearly as a function of the scale-height, with the largest differences being between experiment "G1" and "G5". This is caused by the fast expansion of the magnetic field with height as the scale-height is decreased. Furthermore, this decreases the vertical tension forces accelerating the jets upwards and allow them to expand in the horizontal direction even further as they dynamically evolve (see Fig. 1).

By comparing the graphs showing the location of peak jet velocity versus its position along $y$, it is seen that these are easiest to distinguish at late times when the peak jet velocities approach their maximum value, $V_{\mathrm{A} 0}$. This type of plot would have been more useful if the experiments were carried out for much longer time, following the evolution of magnetic reconnection to a steady state. This would, however, have required larger numerical domains, more grid points, and therefore a substantially increased computing time.
By comparing the density and temperature profiles shown in Fig. 6, it is seen that these reveal more distinct variations between the five experiments than the peak jet velocities discussed above. The largest differences in both mass density, $\rho_{\text {jet }}^{*}$, and plasma temperature, $T_{\text {jet }}^{*}$, at the location of the maximum jet velocity are found between experiments "G1" and "G5". The mass density decreases significantly towards $t / t_{\mathrm{A} 0}=1.0$, and then, in most of the cases, it is found to increase strongly afterwards. The exception to this behaviour is obtained in experiment "G1", where the mass density is maintained at a low level. For the temperature, it is seen that in experiment "G5" an initial increase is followed by a rapid decrease towards a slowly changing temperature after time $t / t_{\mathrm{A} 0}=1.5$. The direct opposite behaviour is observed in experiment "G1", where the temperature increase occurs at later times, altered by a slight decrease towards $t / t_{\mathrm{A} 0}=5.0$. This result shows that the stratification has a significant influence 

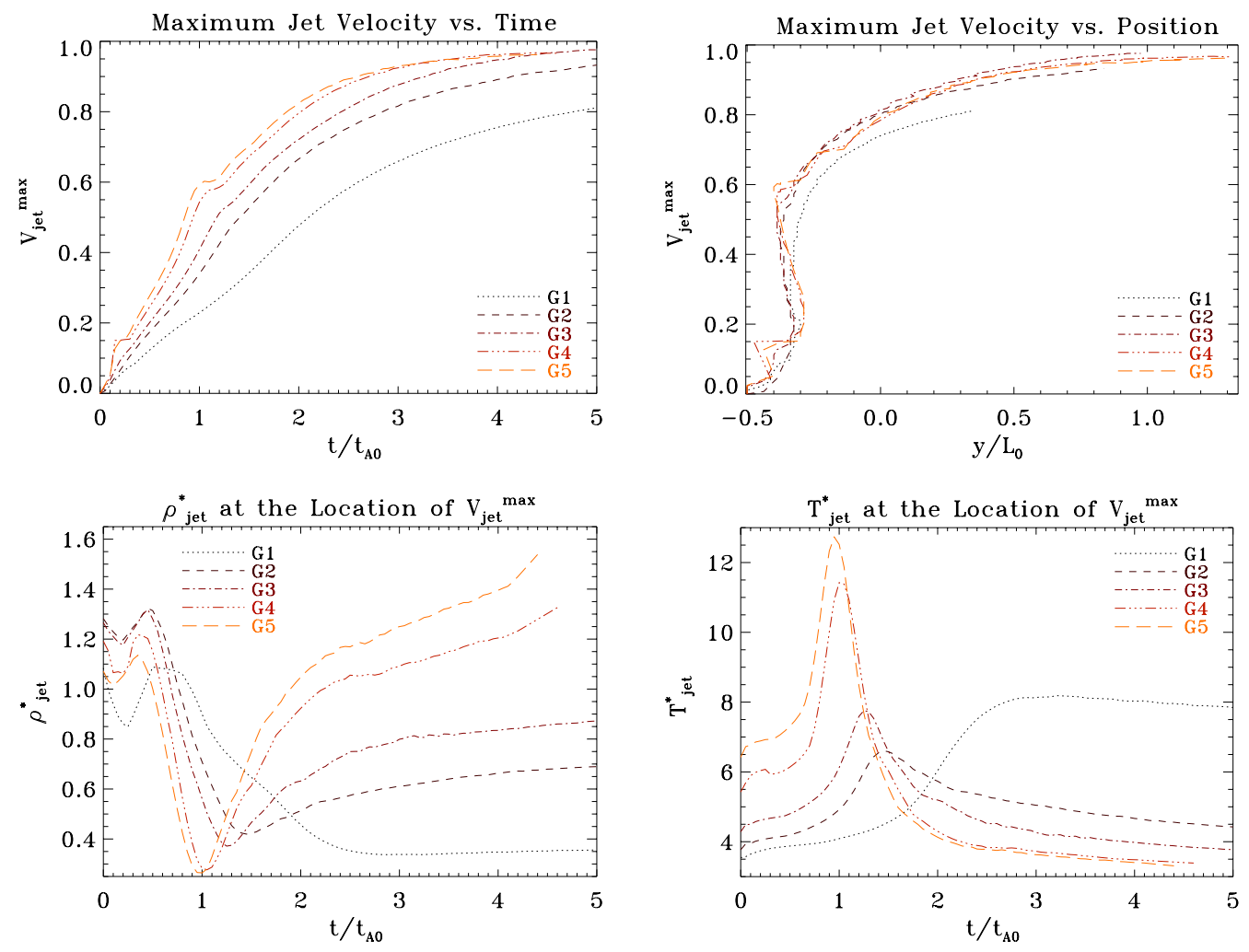

Fig. 6. The maximum flow velocity of the "blue" jet is shown as a function of time (top left) and position along the $y^{*}-$ axis (top right) for all five experiments. In the top right panel time is measured along the curves starting from the lower left corner. The mass density (bottom left) and temperature (bottom right) at the location of maximum jet velocity are also shown as functions of time.

on $\rho_{\text {jet }}^{*}$ and $T_{\text {jet}}^{*}$, with a large scale-height providing the largest time-dependent changes in these parameters.

As already mentioned earlier, the thermal energy inside the reconnection jets is nearly uniform. This implies that changes in the plasma temperature are reciprocal to those in the mass density. This relationship is clearly seen by comparing the two bottom panels in Fig. 6 .

Finally, Table 4 shows the width of the shocks as a function of the scale-height measured at time $t^{*}=4.4$. Two measurements for each jet are included. The first one (Bow shock) measures the width of the head of the reconnection jet (where the slow-mode shocks terminate), while the second one (Slow shock) represents the maximum jet width measured between the two standing slowmode shocks. From this, it is seen that the maximum width of the "blue" jet scales inversely with the scaleheight, while the maximum width of the "red" jet is unaffected by the change in the scale-height. Instead, by looking at the width of the bow shocks, it can be seen that the width of both the "red" and "blue" jets changes as the scale-height is varied. Both widths increase as the scaleheight is decreased. This is surprising since the field lines are converging towards the $x^{*}=0$ axis as the "red" jet propagates towards the lower boundary. One would therefore expect the opposite behaviour, i.e., a decrease in the width of the "red" jet as the scale-height is decreased. It has to be mentioned, however, that these measurements are made at the same physical time, but the jets in the various experiments have advanced quite differently from the origin at this time. The difference in the vertical propagation of the jets can easily be seen in Fig. 1, where experiments "G1" and "G5" are compared. If, instead, one measures the jet width at the same distance from the reconnection site, the differences between the experiments would be even larger. This indicates that the magnetic field structure has a significant influence on the jet expansion as the jets move into an expanding magnetic field.

\section{Summary and future work}

We have examined the dynamical consequences of magnetic reconnection in a $2 \mathrm{D}$ stratified environment representing the "quiet" Sun transition region. The inclusion of gravity was found to have a non-trivial role in determining the initial magneto-hydrostatic equilibrium. An analytical solution that allows for an exponential dependence of the initial physical conditions with height has been found.

Five different initial states have been investigated. These were constructed by assigning different values for the scale-height. This way, the initial physical states covered solutions ranging from a cool 2D current concentration surrounded by hotter plasma (experiment "G1"; small scale-height), to a hot current structure surrounded by cooler ambient plasma (experiment "G5"; large 
Table 4. Width of the jets at $t^{*}=4.4$.

\begin{tabular}{|cccccc|}
\hline Experiment & G1 & G2 & G3 & G4 & G5 \\
\hline \hline "red" jet - Slow shock & 0.487 & 0.487 & 0.487 & 0.487 & 0.487 \\
\hline "red" jet - Bow shock & 0.183 & 0.167 & 0.151 & 0.143 & 0.135 \\
\hline "blue" jet - Slow shock & 0.653 & 0.591 & 0.556 & 0.504 & 0.487 \\
\hline "blue" jet - Bow shock & 0.215 & 0.183 & 0.167 & 0.151 & 0.135 \\
\hline
\end{tabular}

scale-height). In all cases, the initial plasma temperature is an increasing function of height. The relative changes in temperature across the current structure depend on the assumed scale-height, thus determining the initial "hot/cold" current sheet solutions.

In all experiments, magnetic reconnection was driven in an ad hoc manner, through a localised magnetic diffusion being induced in the $2 \mathrm{D}$ current structure from time zero. At early phases of the reconnection process, we discovered a complicated double-oscillatory ("chess-mate" like) pattern of the vorticity. This is caused by initial deviations in the force balance, since the magnetic field is diffused away faster than the pressure gradient reacts on changes in the Lorentz force. As a result, the Lorentz force gets a double-wave pattern in the horizontal $(x)$ direction. This combined with the very initial compressing effect of the Lorentz force in the vertical $(y)$ direction, produces a complex pattern of the vorticity. The alternating signs of the vorticity were found to follow those of the Lorentz forces with respect to the $x^{*}$ and $y^{*}$ coordinates. This initial phase can only survive as long as the diffusive part of the induction equation dominates the dynamics of the diffusion region, i.e., when the magnetic Reynolds number is less than unity. As soon as advection of material from outside this region is initiated, the complex vorticity pattern is suppressed and eventually vanishes. This occurs at about the time when the advective term in the induction equation becomes comparable in magnitude to the diffusive term. By comparing the five different experiments, it was found that the lifetime of this phase scales inversely with the scale-height. One important reason for this is that the sound speed in the diffusion region becomes low relative to the speed of magnetic diffusion as the scale-height is decreased. Thus, it takes longer time for the pressure gradient to readjust to changes in the Lorentz force imposed by the locally enhanced magnetic diffusivity.

It has been found that there is a number of factors that influence the dynamic time-scales on which the two reconnection driven jets evolve. In the first place, there is a difference in the magnetic tension forces in the positive and negative $y$-directions, with a predominance of the tension force in the negative direction. Secondly, the effect of gravity as an external force in the momentum equation is to help accelerate the "red" jet further, while decelerate the "blue" one. On the other hand, the "red" jet is slowed down by the fact that the mass density exponentially increases in the negative $y$-direction. All these factors taken together have a strong impact on the time-dependent evolution of the flow velocities in the two jets.

In our numerical experiments, the differences in the initial magnetic structure determined by the scale-height, $c$, were found to have important implications for the evolution of reconnection event. For large values of $c$ parameter, the magnetic field structure is nearly independent of height and the two outflow jets appear almost identical. When the scale-height, $c$, is decreased, the magnetic field expands away from the current "sheet" and its strength decreases with height. This results in an asymmetry between the two jets, with the upward propagating jet expanding much more in the horizontal direction than that moving downwards. The faster the magnetic field strength decreases with height, the further the reconnection jets expand in the horizontal direction. We have also found that the vertical jet velocities decrease as the scale-height is decreased.

Although the external force of gravity has been taken into account in constructing the initial magnetohydrostatic configurations, these are still unrealistic as applied to the Sun. This is mostly due to the fact that these models represent a situation where the plasma $\beta$ increases with height. This is one disadvantage resulting from the purely 2D MHD modelling involved here. An extension of the model to $2.5 \mathrm{D}$, however, could in principle change the behaviour of the plasma $\beta$ into a decreasing function with height. Such an extension of the model should be investigated.

In the following, assume the blue- and red-shifted Doppler components observed during solar explosive events are due to bi-directional outflow jets, like the ones examined here. The physical parameters that can be derived from related observations are the velocities of Doppler shifted components and the emission measure. One could then directly relate these Doppler velocities with the jet velocities to within a projection factor. Thus, a more detailed comparison between the velocities of "red" and "blue" jets may provide essential information about the fine structure of magnetised plasma in which the reconnection event takes place. Furthermore, for optically thin emitting plasma, the emission measure represents the integrated density square along the line-of-sight. By assuming that the emission measure of reconnection jets 
may be determined, then its temporal variations could indicate changes in the physical conditions in which these jets evolve. Information about the temporal variations in plasma temperature is more difficult to obtain from observational point of view. Recently, TRACE data on the ratio between two well chosen spectral lines observed nearly simultaneously have been used to derive temperature profiles along loops (Aschwanden et al. 1999). On the other hand, Schmelz et al. (2001) reported problems with this approach and appealed that more advanced methods are required for getting reliable temperature estimates for coronal plasmas. Ideally speaking, the combination of such observational results with a family of reconnection solutions could help us better understand the functional behaviour of magnetised plasma in which the reconnection process takes place. This is, however, only true if the numerical models represent sufficiently realistic situations, unlike the simulations presented here.

In a follow-up investigation, we intend to synthesise resonance transitions of Li-like ions, namely C IV $1548 \AA$, O VI $1032 \AA$, and Ne VIII $770 \AA$, based on the MHD experiments presented here. This is done in order to examine the observable consequences of $2 \mathrm{D}$ magnetic reconnection in a stratified physical environment for the purposes of studying solar explosive events.

In conclusion, we believe that the results presented here give a physical insight to the dynamical consequences of magnetic reconnection in a "quiet" solar transition region environment. Although our model investigations involved 2D MHD, they revealed a high degree of complexity in the physical conditions produced by an ongoing magnetic reconnection. In the future, we are challenged to further explore this physical process and its implications, since these are of fundamental importance in understanding the dynamic nature of the Sun.

Acknowledgements. The National Center for Atmospheric Research (NCAR) is sponsored by the National Science Foundation. Research at Armagh Observatory is grant-aided by the N. Ireland Dept. of Culture, Arts and Leisure, while partial support for software and hardware is provided by the STARLINK Project which is funded by the UK PPARC.
K. Galsgaard was supported by PPARC in the form of an Advanced Fellowship. I. Roussev dedicates his studies to Ana and Elena. He is also thankful to the Department of Applied Mathematics, University of St. Andrews, and the NSO in Tucson, Arizona, for the support provided during his visits there. The MHD experiments were carried out on the PPARC funded Compaq MHD Cluster in St. Andrews. We are all very thankful to B. C. Low, Tom Bogdan, Eric Priest, Gerry Doyle, Philip Judge, Tom Holzer, and an unknown referee for the useful comments and suggestions made while this manuscript was prepared.

\section{References}

Aschwanden, M. J., Fletcher, L., Schrijver, C. J., \& Alexander, D. 1999, ApJ, 520, 880

Chen, P. F., Fang, C., Tang, Y. H., \& Ding, M. D. 1999 ApJ, 513,516

Dere, K. P., Bartoe, J.-D. F., \& Brueckner, G. E. 1991, J. Geophys. Res., 96, 9399

Forbes, T. G., Priest, E. R. \& Hood, A. W. 1982, J. Plasma Phys., 27(1), 157

Forbes, T. G., \& Priest, E. R. 1984, Sol. Phys., 94, 315

Innes, D. E., Inhester, B., Axford, W. I., \& Wilhelm, K. 1997, Nature, 386, 811

Krucker, S., Benz, A. O., Bastian, T. S., \& Acton, L. W. 1997, ApJ, 488, 499

Nordlund, Å., \& Galsgaard, K. 1995, A 3D MHD Code for Parallel Computers, www.astro.ku.dk/ klaus/

Pike, C. D., \& Mason, H. E. 1998, Sol. Phys., 182, 333

Priest, E. R., \& Forbes, T. G. 1986, J. Geophys. Res., 91, 5579

Priest, E. R. 1998, Astro. Space Sci., 264, 77

Roussev, I., Galsgaard, K., Erdélyi, R., \& Doyle, J. G. 2001a, A\&A, 370, 298

Roussev, I., Galsgaard, K., Erdélyi, R., \& Doyle, J. G. 2001b, A\&A, 375, 228

Roussev, I., Doyle, J. G., Galsgaard, K., \& Erdélyi, R. 2001c, A\&A, 380, 719

Schmelz, J. T., Scopes, R. T., Cirtain, J. W., Winter, H. D., \& Allen, J. D. 2001, ApJ, 556, 896

Ugai, M., \& Tsuda, T. 1977, J. Plasma Phys., 17(3), 337

Wilhelm, K., Innes, D. E., Curdt, W., Kliem, B., \& Brekke, P. 1998, ESA SP, 421, 103

Wilhelm, K. 2000, A\&A, 360, 351

Yokoyama, T., \& Shibata, K. 1996, PASJ, 48, 353 